Lett. 21, 1476 (1968); R. A. Bryan, Phys. Rev. D 10, 3853 (1974); R. A. Bryan and A. Gersten, Phys. Rev. Lett. 26, 1000 (1971), and 27, 1102(E) (1971); J. Binstock, R. A. Bryan, and A. Gersten, to be published; C. Chiang, R. J. Gleiser, M. Huo, and R. P. Saxena, Phys. Rev. 177, 2167 (1969); J. Binstock, R. Bryan, and A. Gersten, Phys. Lett. 48B, 77 (1974); T. S. Bathia et al., Phys. Rev. Lett. 48, 227 (1982); J. Bystricky, F. Lehar, and P. Winternitz, "On the Status of Time Reversal Invariance in Nucleon-Nucleon Scattering", to be published; H. E. Conzett, in Polarization Phenomena in Nuclear Physics - 1980, edited by G. G. Ohlsen, AIP Conference Proceedings No. 69 (American Institute of Physics, New York, 1981), p. 1422; S. I. Vigdor, in Polarization Phenomena in Nuclear Physics - 1980, edited by G. G. Ohlsen, AIP Conference Proceedings No. 69 (American Institute of Physics, New York, 1981), p. 1429; E. Aprile et al., Phys. Rev. Lett. 47, 1360 (1981).

${ }^{2}$ See Conzett, Ref. 1 .
${ }^{3}$ See Conzett, Ref. 1, and Vigdor, Ref. 1.

${ }^{4}$ P. L. Csonka and M. J. Moravesik, Phys. Rev. 152, 1310 (1966). See also Bryan and co-workers, Ref. 1; Handler, Ref. 1; Bathia et al., Ref. 1.

${ }^{5} \mathrm{An}$ exception is the discussion of time-reversal invariance for the reaction $0+\frac{1}{2} \rightarrow 0+\frac{1}{2}$ in R. J. BlinStoyle, Fundamental Interactions and the Nucleus (North-Holland, Amsterdam, 1973), pp. 216-218, where it is mentioned in passing that the derivation assumes both time-reversal invariance and rotation invariance.

${ }^{6}$ G. R. Goldstein and M. J. Moravcsik, "Polarization Experiments and the Isotropy of Space", to be published.

${ }^{7}$ M. J. Moravesik, in Recent Developments in Particle Physics, edited by M. Moravcsik (Gordon and Breach, New York, 1966), p. 207, theorem 5.

${ }^{8}$ P. L. Csonka, M. J. Moravesik, and M. D. Scadron, Ann. Phys. (N.Y.) 40, 100 (1966).

${ }^{9}$ P. L. Csonka, M. J. Moravesik, and M. D. Scadron, Phys. Rev. Lett. 14, 861 (1965).

\title{
Experimental Tests of Higher-Order Quantum Electrodynamics at Small Distances
}

B. Adeva, D. P. Barber, U. Becker, G. D. Bei, J. Berdugo, G. Berghoff, A. Böhm, J. G. Branson, D. Buikman, J. D. Burger, M. Capell, M. Cerrada, C. C. Chang, H. S. Chen, M. Chen, M. L. Chen, M. Y. Chen, C. P. Cheng, R. Clare, E. Deffur, P. Duinker, Z. Y. Feng, H. S. Fesefeldt, D. Fong; M. Fukushima, J. C. Guo, A. Hariri, D. Harting, T. Hebbeker, G. Herten, M. C. Ho, M. M. Ilyas, D. Z. Jiang, D. Kooijman, W. Krenz, Q. Z. Li, D. Luckey, E. J. Luit, C. Maña, G. G. G. Massaro, T. Matsuda, H. Newman, M. Pohl, F. P. Poschmann, J. P. Revol, M. Rohde, H. Rykaczewski, A. Rubio, J. Salicio, I. Schulz, K. Sinram, M. Steuer, G. M. Swider, H. W. Tang, D. Teuchert, Samuel C. C. Ting, K. L. Tung, F. Vannucci, M. White, S. X. Wu, T. W. Wu, and R. Y. Zhu

III. Physikalisches Institut, Technische Hochschule Aachen, D-5100 Aachen, Fedeval Republic of Germany, and Deutsches Elektronen-Synchrotron DESY, D-2000 Hamburg 52, Federal Republic of Germany, and Laboratory for Nuclear Science, Massachusetts Institute of Technology, Cambridge, Massachusetts 02139, and Junta de Energia.Nuclear, Madrid, Spain, and Nationaal Instituut voor Kernfysica en Hoge-Energiefysica, Amsterdam, The Netherlands, and Institute of High Energy Physics, Chinese Academy of Science, Peking, People's Republic of China (Received 28 January 1982)

A direct test is presented of higher-order QED $\left(\alpha^{4}\right)$ at large momentum transfers (up to $\sim 100 \mathrm{GeV}^{2}$ ). These tests were carried out with the MARK-J detector at PETRA by comparing the measured cross section for the process $e^{+} e^{-} \rightarrow e^{+} e^{-} \mu^{+} \mu^{-}$with the prediction of QED for $12 \mathrm{GeV} \leqslant \sqrt{s} \leqslant 36.7 \mathrm{GeV}$. The cross sections and the various kinematic distributions agree with $Q E D$.

PACS numbers: $12.20 . \mathrm{Fv}, 13.10 .+\mathrm{q}$

There have been many tests of QED processes over the last two decades, and they can be grouped into two classes ${ }^{1}$ :

(a) Precision tests of QED such as the anomalous magnetic moment $(g-2)$ of muons and electrons. These processes test higher-order QED at small momentum transfers. (b) High-energy processes such as photoproduction of lepton pairs, Bhabha scattering, and muon-pair production from $e^{+} e^{-}$colliding beams. These processes test first-order QED at large momentum transfers and small distances.

We present new results from an experiment which is a combination of both classes and which 
tests higher-order QED $\left(\alpha^{4}\right)$ at very large momentum transfers. This experiment enables us to probe for the first time higher-order QED theory at very small distances.

The first measurements of lepton pair production from the two-photon process were performed at VEPP II and $\mathrm{ADONE}^{2}$ between 1970 and 1974. Since the first observation of the two-photon process at PETRA, ${ }^{3,4}$

$$
e^{+} e^{-} \rightarrow e^{+} e^{-} \mu^{+} \mu^{-} \text {, }
$$

the accelerator has reached a center-of-mass energy of $\sqrt{s}=36.7 \mathrm{GeV}$, and the total integrated luminosity has increased by a factor of $20\left(\int L d t\right.$ $\left.\approx 30000 \mathrm{nb}^{-1}\right)$. The two-photon process $e^{+} e^{-}$ $\rightarrow e^{+} e^{-} \mu^{+} \mu^{-}$has a large total cross section at PETRA energies $(\sigma \approx 120 \mathrm{nb}$ at $\sqrt{s}=30 \mathrm{GeV})$ and it thus allows for a direct test of QED to order $\alpha^{4}\left(\alpha \approx \frac{1}{137}\right) .^{5}$ The MARK-J detector and the methods of detection of muons, electrons, and photons have been described previously. ${ }^{6}$

Most of the events from reaction (1) occur at small $q^{2}$ so that the electron and the positron scatter at small angles with respect to the beam axis. ${ }^{7}$ For this reason it is usually not possible to observe all four particles in the final state, and we have classified the observed final states in four categories: (a) $\mu \mu$ observed (972 events), (b) $\mu e$ observed (151 events), (c) $e \mu \mu$ observed, (d) ee $\mu \mu$ observed [(c) and (d) combined, 70 events].

In case (a) the two muons and the beam axis tend to be coplanar because the electrons are emitted close to the beams and because of transverse momentum balance. In general, the muons are not collinear and their momentum spectrum is peaked at small values in contrast with the muon pairs from the one-photon annihilation channel.

The event selection is performed by requiring two muons penetrating the detector iron with $P_{t}$ $z 1.5 \mathrm{GeV}$ for at least one of the two muons; $E_{\mu}$ of each muon $<50 \% E_{\text {beam }} ; E_{\text {elm }}<20 \% E_{\text {beam }}$, where $E_{\text {elm }}$ is the amount of electromagnetic energy measured in the shower counters. Figure 1(a) shows that the observed cross section for muon pairs agrees well with the Monte Carlo prediction of QED where, as in all of this study, we have used the Monte Carlo program of Vermaseren $^{8}$ to calculate the predictions of QED for two-photon processes to order $\alpha^{4}$, Higher-order radiative effects have been estimated by Defrise et $a l .{ }^{8}$ to be $\sim 10 \%$ and have not been included in the Monte Carlo program. The data have been
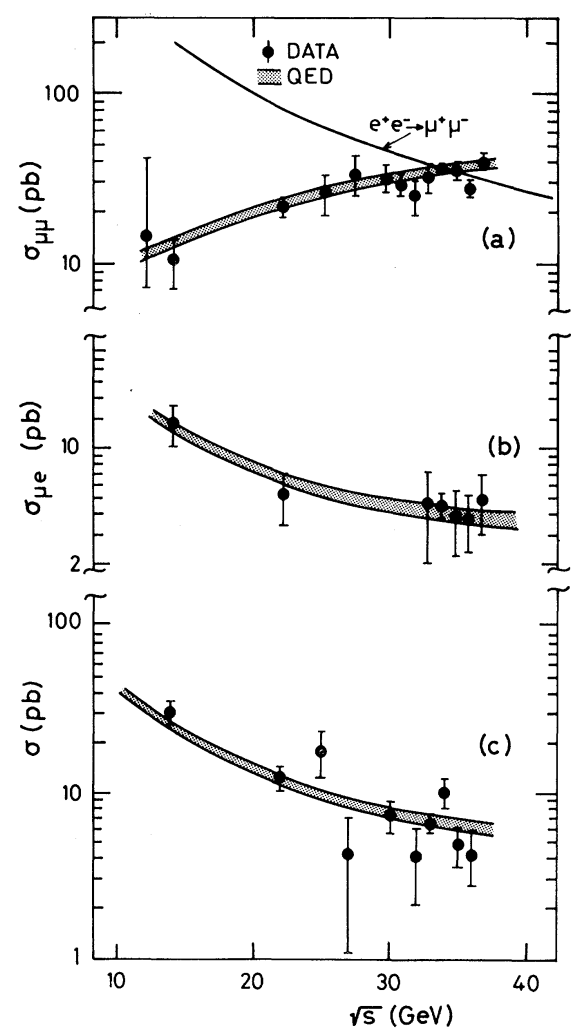

FIG. 1. Comparison of observed cross sections from the reaction (1) with QED as a function of energy $(\sqrt{s})$. The shaded area indicates the accuracy of the Monte Carlo simulation. (a) The case when only $\mu^{+} \mu^{-}$are observed. Also shown is the cross section of $e^{+} e^{-}$ $\rightarrow \mu^{+} \mu^{-}$. (b) The data when $\mu e$ are observed in the final state. (c) The observed cross section for the combined processes of reaction (1) ( $\mu \mu e$ or $\mu \mu e e$ observed) and $e^{+} e^{-} \rightarrow \mu^{+} \mu^{-} \gamma$.

corrected for background from $e^{+} e^{-} \rightarrow\left(\tau^{+} \tau^{-}, \mu^{+} \mu^{-}\right)$. This correction is $-3.5 \%$ at the highest and $-2.8 \%$ at the lowest energies. Our Monte Carlo study shows that for the undetected electrons the average $q^{2} \approx-0.2 \mathrm{GeV}^{2}$.

The selection for case (b) is defined by one muon reaching the outside drift chambers; $E_{\mathrm{elm}}$ $>20 \% E_{\text {beam }}, 16^{\circ}<\theta_{\mathrm{el} \mathrm{m}}<30^{\circ}$, or $150^{\circ}<\theta$ elm $<164^{\circ}$, $\xi>30^{\circ}$, where $\theta_{\text {elm }}$ is the angle between the electron momentum and the beam axis and $\xi$ is the acollinearity angle between the muon and the observed electron or positron. With these cuts the background from $e^{+} e^{-} \rightarrow\left(\mu^{+} \mu^{-} \gamma, \tau^{+} \tau^{-}, e^{+} e^{-} \tau^{+} \tau^{-}\right.$, $e^{+} e^{-}+$hadrons) is $7 \%$ at $\sqrt{s}=35 \mathrm{GeV}$ and $13 \%$ at $\sqrt{s}=14 \mathrm{GeV}$, and has been corrected from the data. Figure 1(b) shows the measurement of the observed cross section compared with the prediction of QED.

In cases (c) and (d) and $\mu \mu \gamma$ final states, we 

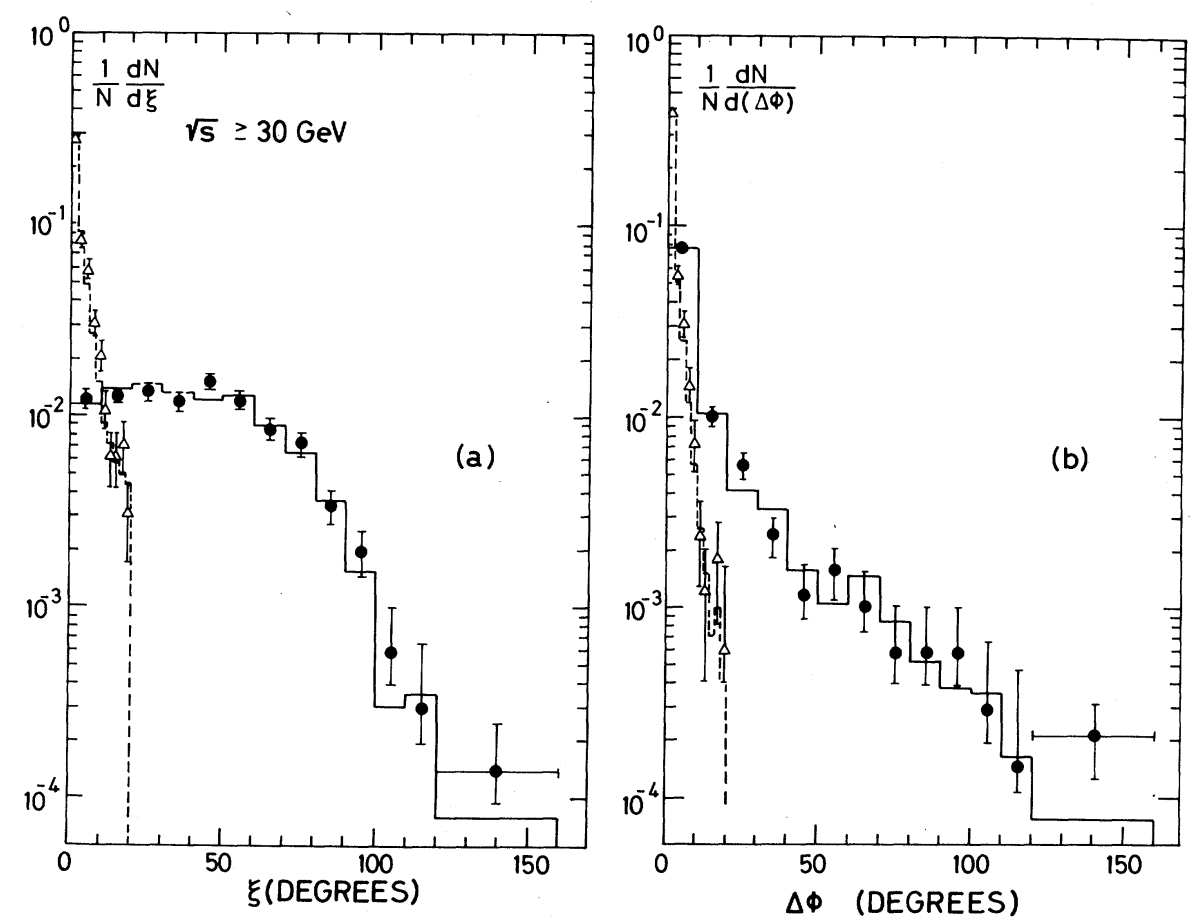

FIG. 2. Angular distributions (circles) between muons from reaction (1) and comparison with QED predictions (solid line). Also shown are angular distributions (triangles) and predictions from QED (dashed line) for muons from $e^{+} e^{-} \rightarrow \mu^{+} \mu^{-}$. (a) The acollinearity distribution; (b) the acoplanarity distribution.

require two muons to penetrate the iron with at least one muon reaching the outer chambers. Further conditions are

$$
E_{\mathrm{elm}}>20 \% E_{\text {beam }} \text { and } 12^{\circ}<\theta_{\text {elm }}<168^{\circ} \text {. }
$$

Figure 1(c) shows the measurement of the combined cross section for $\mu \mu \gamma$ events and for events in classes (c) and (d). The measurements are compared with the predictions of QED. Although the cross sections (c) and (d) are small compared with the radiative process $\mu \mu \gamma$, the $\mu \mu e$ events can be distinguished from $\mu \mu \gamma$ by the requirement that the missing momentum is $\geqslant \frac{1}{4} E_{\text {beam }}$ and $E_{\mu} \leqslant 50 \% E_{\text {beam }}$.

We have made a detailed study of the kinematic properties of muon pairs from class (a) for $\sqrt{s}$ $\geqslant 30 \mathrm{GeV}$. In all cases there is very good agreement with the prediction of QED for the two-photon process. In Figs. 2(a) and 2(b) we show the comparison of acollinearity and acoplanarity distributions for the muon pairs from class (a) and from $e^{+} e^{-}-\mu^{+} \mu^{-}$with predictions of QED.

The invariant-mass $(M)$ distribution of the muon pair [Fig. 3(a)] does not show any obvious structure to indicate a new state of charge parity $C=+1$ decaying into a muon pair. The quantity $d \sigma / d \boldsymbol{P}_{t}^{2}[$ Fig. $3(\mathrm{~b})]$, where $\boldsymbol{P}_{t}$ is the transverse momentum of the muon with respect to the beam line, is of particular interest, partially because it is a test of hard-scattering models ${ }^{9}$ and also because it might be expected to agree with the distribution of the produced quark transverse momentum in two-photon production via $e^{+} e^{-}$ $\rightarrow e^{+} e^{-} q \bar{q} .{ }^{10}$ We have fitted our measurement with the form $A P_{t}^{-B}$ for $P_{t}{ }^{2}>3.2 \mathrm{GeV}^{2}$, resulting in $A=8.3 \pm 1.3 \mathrm{nb} / \mathrm{GeV}^{2}$ and $B=4.9 \pm 0.2$, with $\chi^{2}$ $=17.0$ and for 16 degrees of freedom. That this measurement departs from a well known and expected $P_{t}{ }^{-4}$ behavior ${ }^{9}$ is supported by the QED Monte Carlo calculation, which also disagrees with $P_{t}{ }^{-4}$ but reproduces the data quite closely $\left(\chi^{2}=21\right.$ for 18 degrees of freedom for $P_{t}{ }^{2}>3.2$ $\left.\mathrm{GeV}^{2}\right)$. Indeed, a careful study of the $P_{t}$ dependence indicates that for these center-of-mass energies the slope parameter $B$ depends upon $\sqrt{s}$ and $M$, and approaches 4 only in the limit $s \rightarrow \infty$.

It is interesting to note that Figs. 3(a) and 3(b) show that QED is correct to $\alpha^{4}$ and up to $M^{2}$ $\approx 100 \mathrm{GeV}^{2}$ and $P_{t}{ }^{2} \approx 50 \mathrm{GeV}^{2}{ }^{2}$ In cases (b), (c), and (d), the requirement of one electron or positron at large angle $\theta \approx 16^{\circ}$ allows the study of a domain of phase space where one of the two pho- 

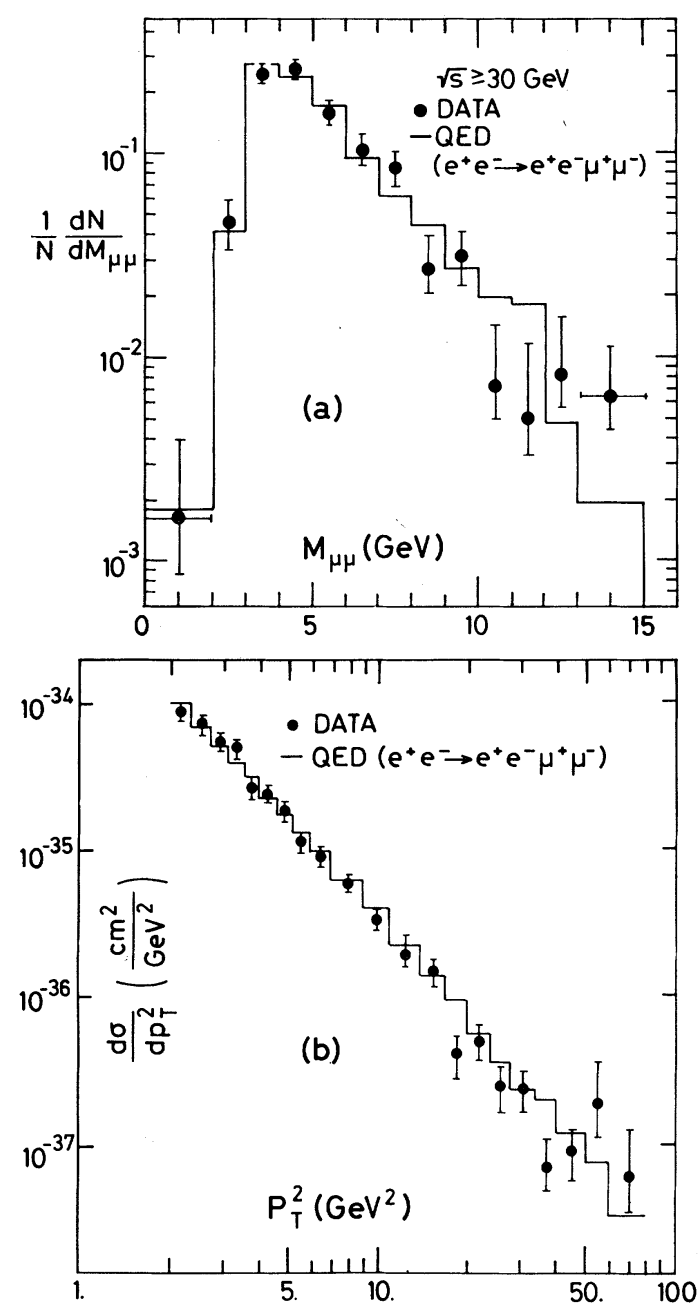

FIG. 3. (a) Invariant-mass distribution for muon pairs from the data of case (a). (b) $d \sigma / d P_{t}{ }^{2}$ as a function of $P_{t}^{2}$ for the sample obtained with use of one entry for each muon. The lines are the QED calculation.

tons exchanged is far off the mass shell. In this case $\left\langle q^{2}\right\rangle \simeq 33 \mathrm{GeV}^{2}$ for the electron recoiling at large angle.

In conclusion, the properties of the two-photon process $e^{+} e^{-} \rightarrow e^{+} e^{-} \mu^{+} \mu^{-}$have been found to agree well with the prediction of QED to order $\alpha^{4}$ over a large momentum-transfer range. In our kinematical region, the quantity $d \sigma / d P_{t}{ }^{2}$ is proportional to $P_{t}{ }^{-4.9 \pm 0.2}$.

We wish to thank Professor V. Soergel, Professor H. Schopper, Professor E. Lohrmann, Professor G.-A. Voss, Dr. F. J. Eppling, and Dr. G. Söhngen for their valuable support. We acknowledge helpful discussions with Professor K. Huang, Professor E. Picasso, Dr. J. A. M. Vermaseren, Dr. A. Courau, M. Paranjape, and $\mathrm{N}$. Wermes. We also thank P. Berges, S. Burger, H. Geller, S. Marks, and D. Osborne for their technical and administrative help. This work was supported by the Deutsches Bundesministerium für Forschung und Technologie.

${ }^{1}$ J. G. Asbury et al., Phys. Rev. Lett. 18, 65 (1967); H. Alvensleben et al., Phys. Rev. Lett. $\overline{21}, 1501$ (1968); V. Alles-Borelli et al., Nuovo Cimento A 7 , 345 (1972); H. Newman et al., Phys. Rev. Lett. $32,4 \overline{83}$ (1974); J. E. Augustin et al., Phys. Rev. Lett. 34, 233 (1975); L. H. O'Neill et al., Phys. Rev. Lett. 37, 395 (1976); S. J. Brodsky and S. D. Drell, Annu. Rev. Nucl. Sci. 20, 147 (1970); E. Picasso et al., Phys. Rep. 68, 93 (1981); J. Bailey et al., Phys. Lett. 68B, 191 (1977); M. Pohl, in Proceedings of the Sixteenth Rencontre de Moriond, Les Arcs, France, 1981 (to be published); A. Böhm, Technische Hochschule Aachen, Report No. PITHA 81/ 30, 1981 (to be published); J. G. Branson, in Proceedings of the 1981 Symposium on Lepton and Photon Interactions at High Energies, Bonn, 24-29 August 1981 (to be published).

${ }^{2}$ V. E. Balakin et al., Phys. Lett. $\underline{34 \mathrm{~B},} 99$ (1971); C. Bacci et al., Lett. Nuovo Cimento 3, 709 (1972).

${ }^{3} \mathrm{~A}$. Courau, in Proceedings of the Fourth International Colloquium on Photon-Photon Interactions, Paris, 6-9 April 1981, edited by George W. London (World Scientific, Singapore, 1981).

${ }^{4}$ D. P. Barber et al., Phys. Rev. Lett. 43,1915 (1979); Ch. Berger et al. (PLUTO Collaboration), DESY Report No. 79/65, 1979 (unpublished); W. Wagner, DESY Report No. 80/102, 1980 (unpublished).

${ }^{5}$ D. P. Barber et al., Phys. Rev. Lett. 45, 1904 (1980); J. P. Revol, Ph.D. thesis, Massachusetts Institute of Technology, 1981 (unpublished); M. M. White, $\mathrm{Ph} . \mathrm{D}$. thesis, Massachusetts Institute of Technology, 1981 (unpublished).

${ }^{6}$ The MARK-J Collaboration, Phys. Rep. $\underline{63}, 337$ (1980).

7'Two-photon process bibliography: F. Low, Phys. Rev. 120, 582 (1960); J. C. Le Guillou, C. R. Acad. Sci. 261,326 (1965); H. Terazawa, Rev. Mod. Phys. 45,615 (1973); F. Calogero et al., Phys. Rev. 120, $1860(1960)$; S. J. Brodsky et al., Phys. Rev. Lett. 25 , 972 (1970). Also see Proceedings of the International Workshop on $\gamma \gamma$ Collisions, Amiens, France, 8-12 April 1980 (to be published); A. Courau, Laboratoire de l'Accélérateur Linéaire Report No. LAL/81-03, 1981 (unpublished); R. Brandelik et al., Z. Phys. 107B, 290 (1981); W. Bartel et al ., Phys. Lett. 107B, 163 (1981); Ch. Berger et al., Phys. Lett. 107B, 168 (1981).

${ }^{8}$ All Monte Carlo calculations concerning $e^{+} e^{-}$ $\rightarrow e^{+} e^{-} \mu^{+} \mu^{-}$were made with use of J. A. M. Vermaseren's event generator and a detailed simulation of the MARK-J detector. We also used a Monte Carlo simulation of $e^{+} e^{-} \rightarrow \mu^{+} \mu^{-} \gamma$, correct to order $\alpha^{3}$, based on the recent work described in F. A. Berends and 
R. Kleiss, DESY Report No. 80/66 (unpublished). For higher-order radiative effects, see M. Defrise, $\mathrm{Z}$. Phys. C 9, 41 (1981); M. Defrise, S. Ong, J. Silva, and C. Carimalo, Phys. Rev. D 23, 663 (1981).

${ }^{9}$ S. M. Berman, J. D. Bjorken, and J. B. Kogut,
Phys. Rev. Lett. D 4 , 3388 (1971)。

${ }^{10} \mathrm{H}$. Spitzer, DESY Report No. 80/43, 1980 (unpublished); W. Bartel et al., Phys. Lett. 107B, 163 (1981); R. Brandelik et al., Phys. Lett. 107B, 290 (1981).

\title{
Study of Parity Nonconservation in $p \alpha$ Scattering
}

R. Henneck, Ch. Jacquemart, J. Lang, R. Müller, Th. Roser, M. Simonius, and F. Tedaldi Laboratorium für Kernphysik, Eidgenössische Technische Hochschule, CH-8093 Zürich, Switzerland

and

W. Haeberli

University of Wisconsin, Madison, Wisconsin 53706

and

S. Jaccard

Schweizerisches Institut für Nuklearforschung, CH-5234 Villigen, Switzerland

(Received 30 December 1981)

\begin{abstract}
Parity nonconservation in $p \alpha$ scattering has been studied by comparing the cross sections $\sigma^{+}$and $\sigma^{-}$for longitudinally polarized $46-\mathrm{MeV}$ protons of positive and negative helicity. The longitudinal analyzing power is found to be $A_{z}=(0.3 \pm 1.3) \times 10^{-7}$. This result, together with earlier measurements on parity nonconservation in $p p$ scattering, gives new limits for the weak pion-nucleon coupling constant.
\end{abstract}

PACS numbers: 11.30.Er, 25.40.Cm, 13.75.Gx, 24.70.+s

According to present theory, the interaction between nucleons ( $N N$ interaction) contains a small contribution from weak interactions (hadronic weak currents) which can be described by weakcoupling constants associated with the exchange of various mesons $(\pi, \rho, \omega$, etc.). Experimentally, the weak $N N$ potential can be detected because it leads to a small parity nonconservation in nuclear interactions. The presence of such effects has been clearly demonstrated, e.g., through the detection of circular polarization $P_{\gamma}$ in the $\gamma$ decay of various nuclei, but there is insufficient information at present to determine the individual weak meson-nucleon coupling constants (see Haeberli $^{1}$ and Desplanques ${ }^{2}$ for reviews).

The work reported here is the first measurement of parity nonconservation in $p \alpha$ scattering. The experiment consists of comparison of cross sections $\sigma^{+}$and $\sigma^{-}$for logitudinally polarized proton beams of positive and negative helicity. Recent calculations $\mathbf{s}^{3,4}$ of the longitudinal analyzing power $A_{z}=\left(\sigma^{+}-\sigma^{-}\right) /\left(\sigma^{+}+\sigma^{-}\right)$predict values of the order of a few times $10^{-7}$ and suggest that the contribution from the weak pion-nucleon coupling constant, $f_{\pi}$, should dominate $A_{z}$. The value of $f_{\pi}$ is of particular interest because this coupling constant is strongly affected by the weak neutral currents in the theory of Weinberg and Salam. Study of the $p \alpha$ system is attractive because it is the simplest one available (apart from the $p p$ and and $n p$ systems) and because its scattering states are relatively well known.

The experimental technique was essentially the same as in our earlier investigation on $p p$ scattering. ${ }^{5,6}$ The arrangement is shown schematically in Fig. 1. Protons are scattered in a 100-bar He target whose walls are sufficiently thick $(2-\mathrm{mm}$ $\mathrm{Al}$ alloy) that charged particles from breakup reactions are stopped. Protons scattered by $23^{\circ}-$ $97^{\circ}$ are detected in a hydrogen-filled (1-bar) cyclindrical ionization chamber which surrounds the target. The proton beam was provided by the Swiss Institute for Nuclear Research cyclotron which is equipped with an atomic-beam-type polarized-ion source. The arrangement in Fig. 1 is preceded by a spin-precession solenoid and a $47.6^{\circ}$ deflection magnet, which, together, precess the vertical polarization of the beam from the cyclotron $\left( \pm P_{y}\right)$ into a beam of longitudinal polarization $\pm P_{z}$ or $\mp P_{z}$, depending on the sign of the solenoid field. The helicity of the beam is reversed every $30 \mathrm{msec}$ by switching rf transitions in the ion source. Every few hours the overall phase of $P_{z}$ is reversed by reversal of 